\title{
UNIMODAL TIME-SYMMETRIC DYNAMICS
}

\section{CHRIS BERNHARDT}

\author{
Received 11 February 2002
}

\begin{abstract}
Time-symmetric cycles are defined. Unimodal time-symmetric cycles are classified. A one-parameter family of maps of the closed unit interval that exhibits this classification is given.
\end{abstract}

2000 Mathematics Subject Classification: 37E05.

1. Introduction. In one-dimensional dynamics, the maps that are studied are usually noninvertible. The initial reason for studying these maps was that they had complicated behavior that was reminiscent of diffeomorphisms of two-dimensional spaces and differential equations in three dimensions. It is surprising that one-dimensional maps seem to model some physical systems since physical systems are symmetric in time and most one-dimensional models are noninvertible and so cannot be run backwards. In this paper, we begin the study of modelling invertible data using unimodal maps.

Unimodal maps have been used as models of physical systems (see, e.g., [6]). In the unimodal case, there is a linear ordering of periodic orbits that is most easily described in terms of the orbits cycle type. Thus, an orbit with cycle type (13425) occurs between a fixed point with cycle type (1) and the period three orbit of cycle type (123). If we take the standard map $f_{\mu}=\mu x(1-x)$, then, as the parameter $\mu$ is increased, we see first a stable fixed point, then a stable orbit corresponding to the five-cycle, and later a stable orbit corresponding to the three-cycle. Now, imagine these three orbits as corresponding to physical data. If we reverse time the orbits will correspond to the cycles (1), (15243), and (132). However, the ordering of these three cycles is not the same as before. Now, (132) lies between (1) and (15243). (It also should be noted that (15243) is not a unimodal cycle.) In a sense that will be made clear, taking the inverses of the cycles (1) and (123) does not affect their position in the ordering, but (15243) and (13425) are in different positions. Since physical data should not look more complicated if we reverse time, it would seem that we should only observe orbits whose cycles give isomorphic dynamics when we take inverses.

In this paper, we first formalize the notion of these time-symmetric cycles. Then, we completely classify these cycles in the unimodal case. Then, we give an example of a one-parameter family of maps of the interval that exhibits this classification. 
2. Basic ideas. Continuous maps of the interval induce a partial order on the set of cyclic permutations (cycles).

Suppose that a map of the interval has a periodic orbit $P=\left\{p_{1}, \ldots, p_{n}\right\}$, labelled so that $p_{1}<\cdots<p_{n}$. Then, $P$ has the cycle type $\pi$ if $\pi$ is a cycle with the property that $f\left(p_{i}\right)=p_{j}$ if and only if $\pi(i)=j$. A cycle $\pi$ forces a cycle $\mu$, written $\pi \geq \mu$, if every continuous map of the interval that has a periodic point of the cycle type $\pi$ also has a periodic point with the cycle type $\mu$. Baldwin [2] showed that the forcing relation was a partial order on the set of cycles.

Given $\beta$, a cyclic permutation of $\{1, \ldots, m\}$, let $L_{\beta}:[1, m] \rightarrow[1, m]$ denote the map defined by $L_{\beta}=\beta$ on $\{1, \ldots, m\}$ and $L_{\beta}$ is linear on each $[i, i+1]$. The nondegenerate intervals with respect to " $L_{\beta}$ is strictly monotone on $I$ " are called laps of $L_{\beta}$. The cycle $\beta$ is $n$-modal if $L_{\beta}$ has $n+1$ laps. The cycles (1) and (12) are exceptions and will be considered as being unimodal. Since we will be using maps from the unit interval to itself, we will rescale $L_{\beta}$ to form $I_{\beta}:[0,1] \rightarrow[0,1]$ by defining $I_{\beta}=\mu^{-1} L_{\beta} \mu$ where $\mu(x)=(m-1) x+1$.

These piecewise linear maps are minimal in the sense of the following theorem. (See [5, page 171, Theorem 5].)

THEOREM 2.1. The map $I_{\alpha}$ has a periodic orbit with cycle type $\beta$ if and only if $\alpha$ forces $\beta$.

The structure of the set of cycles ordered by the forcing relation is complicated. The local structure is known: immediately above any cycle lie all doubles of that cycle (see [4]). A cycle $\alpha$ of period $n$ is a double if $n$ is even and for $k=1, \ldots, n / 2, \alpha(2 k-1)$ and $\alpha(2 k)$ are consecutive integers. (In this case, $\alpha$ is a "double" of the permutation of $\{1, \ldots, n / 2\}$ defined by $i \mapsto j$ if $\{\alpha(2 i-1), \alpha(2 i)\}=\{2 j-1,2 j\}$.)

We will say that two cycles $\gamma$ and $\delta$ are dynamically equivalent if there exists a homeomorphism $h$ of the interval such that $I_{\gamma}=h^{-1} \circ I_{\delta} \circ h$.

Let $\sigma_{n}$ denote the cycle of length $n$ defined by $\sigma_{n}(i)=n-i+1$ for $i=1, \ldots, n$. If there is no ambiguity in the value of $n$, we will write $\sigma$ instead of $\sigma_{n}$.

THEOREM 2.2. Let $\alpha$ denote a cycle of length $n$. The only two cycles that are dynamically equivalent to $\alpha$ are $\alpha$ and $\sigma \alpha \sigma$.

Proof. Taking $h(x)=x$ in the first case and $h(x)=1-x$ in the second, it is clear that both $\alpha$ and $\sigma \alpha \sigma$ are equivalent to $\alpha$.

Suppose that $I_{\alpha}=h^{-1} \circ I_{\delta} \circ h$. First, we will assume that $h$ is orientation preserving. Then, since $I_{\alpha}$ has a periodic point of the cycle type $\alpha$, so must $I_{\delta}$. Theorem 2.1 then tells us that $\delta$ must force $\alpha$. A similar argument shows that $\alpha$ forces $\delta$ and since the forcing relation is a partial order we have $\alpha=\delta$.

If $h$ is orientation reversing, then $I_{\sigma \delta \sigma}=(h \circ g)^{-1} \circ I_{\delta} \circ(h \circ g)$ where $g(x)=$ $1-x$. Now, $h \circ g$ is orientation preserving and the above argument shows that $\delta=\sigma \alpha \sigma$. 
Clearly, from a physical viewpoint, $\alpha$ and $\sigma \alpha \sigma$ correspond to interchanging right and left, and we would expect them to be dynamically equivalent.

We will say that a cycle $\alpha$ is time-symmetric if $\alpha^{-1}$ is dynamically equivalent to $\alpha$. Since the only cycles that have the property of being self-inverse are (1) and (12), the definition can be restated as $\alpha$ is time-symmetric if $\alpha^{-1}=\sigma \alpha \sigma$.

In what follows, attention will be restricted to unimodal cycles. These are divided into two classes: those with the first lap increasing, and those with the first lap decreasing (the exceptional cycles (1) and (12) will be considered as belonging to both classes). We will denote the first class as unimax cycles and the second class unimin cycles. It is well known that each class is linearly ordered by the forcing relation (see, e.g., [1]).

3. Unimodal time-symmetric cycles. It is clear that if a time-symmetric cycle is unimodal, then so is its inverse. We begin this section by describing which unimodal cycles have unimodal inverses. It turns out that these are exactly the time-symmetric ones.

LEMMA 3.1. If $\theta$ is a unimax cycle of length $n>2$ with unimodal inverse, then there exists a $k, 1 \leq k \leq n$, such that

$$
\theta(i)= \begin{cases}i+k & \text { if } i \leq n-k, \\ n+1-i & \text { if } i>n-k .\end{cases}
$$

If $\theta$ is a unimin cycle of length $n$ with unimodal inverse, then there exists a $k, 1 \leq k \leq n$, such that

$$
\theta(i)= \begin{cases}n+1-i & \text { if } i \leq k, \\ i-k & \text { if } i>k .\end{cases}
$$

PROOF. We will prove the first statement. The proof of the second statement is similar.

If $\theta$ is a unimax cycle we know that $\theta(n)=1$. Let $\theta(1)=m$ and let $k$ denote the inverse of $n$, that is, $\theta(k)=n$. Since $\theta$ is unimax, we know that $\theta$ is increasing on $\{1, \ldots, k\}$ and decreasing on $\{k, \ldots, n\}$. The result will be proved if we can show that there does not exist an $l$ satisfying $k<l<n$ and $m<\theta(l)<n$. Suppose for a contradiction that such an $l$ exists. Then, we have $1<m<\theta(1)<n$. So $\theta^{-1}(1)=n, \theta^{-1}(m)=1, \theta^{-1}(\theta(l))=l$, and $\theta^{-1}(n)=k$. Since $l>k, \theta^{-1}$ is not unimodal.

LEMMA 3.2. If $n$ is odd, the only unimax cycle with unimodal inverse is $(123 \cdots n)$. If $n>2$ is even, there are two unimax cycles with unimodal inverses: $(123 \cdots n)$ and $(135 \cdots(n-1) 246 \cdots n)$.

Proof. It is straightforward to check that cycles of the form $(123 \cdots n)$ and $(135 \cdots(n-1) 246 \cdots n)$ are unimax and have unimodal inverses. From 
the previous lemma, we know that if $\theta$ is a unimax cycle then

$$
\theta(i)= \begin{cases}i+k & \text { if } i \leq n-k \\ n+1-i & \text { if } i>n-k\end{cases}
$$

We must show that $k$ is either 1 or 2 . Let $m$ denote the positive integer such that $1+m k \leq n<1+(m+1) k$. Then, if we look at the orbit of 1 under repeated iteration by $\theta$, it begins with $\{1,1+k, 1+2 k, \ldots, 1+m k\}$. If $1+m k=n$, then, since $\theta(n)=1$ and $\theta$ is a cycle of length $n$, we must have $k=1$. If $1+m k \neq n$, then $\theta(1+m k)=n-m k$. Clearly, $\theta^{m}(n-m k)=n$. Thus, since $\theta$ is a cycle of length $n$, we must have $n=2 m$ and $k=2$.

Similarly, we can prove the following lemma for unimin cycles.

LEMMA 3.3. If $n$ is odd, the only unimin cycle with unimodal inverse is $(1 n(n-1)(n-2)(n-3) \cdots 2)$. If $n>2$ is even, there are two unimin cycles with unimodal inverses: $(1(n-1)(n-2)(n-3) \cdots 1)$ and $(1 n(n-2)(n-4) \cdots 2(n-$ 1) $(n-3) \cdots 3)$.

To simplify the notation let $[n]$ denote $(123 \cdots n)$ and $[n]^{-1}$ denote $(1 n(n-$ $1) \cdots 2)$. The cycle $(135 \cdots(n-1) 246 \cdots n)$ is a double of $[n / 2]$ and will be denoted $[2 * n / 2]$. Finally, $[2 * n / 2]^{-1}$ denotes $(1 n(n-2)(n-4) \cdots 2(n-$ 1) $(n-3) \cdots 3)$.

Since we know that any time-symmetric unimodal cycle will have a unimodal inverse, it is clear that time-symmetric cycles must have the form given in Lemmas 3.2 and 3.3. However, it is straightforward to check that $\sigma[n] \sigma=$ $[n]^{-1}$ and that $\sigma[2 * k] \sigma=[2 * k]^{-1}$. So, every cycle given in Lemmas 3.2 and 3.3 is time-symmetric. Thus, we have the following theorem.

THEOREM 3.4. For every positive integer $n$, the cycles $[n],[n]^{-1},[2 * n]$, and $[2 * n]^{-1}$ are unimodal and time-symmetric. Conversely, every unimodal, time-symmetric cycle is of one of these four types.

4. Forcing and symmetric forcing. The forcing relation for cycles has been studied by many authors. For a good general reference, see [1]. Let $\alpha<\beta$ if the cycle $\beta$ forces $\alpha$.

THEOREM 4.1. Unimodal time-symmetric cycles are ordered by the forcing relation in the following ways: [1] $<[2]<[2 * 2]<[3]<[2 * 3]<\cdots<[k]<$ $[2 * k]<[k+1]<[2 *(k+1)]<\cdots$ and $[1]^{-1}<[2]^{-1}<[2 * 2]^{-1}<[3]^{-1}<$ $[2 * 3]^{-1}<\cdots<[k]^{-1}<[2 * k]^{-1}<[k+1]^{-1}<[2 *(k+1)]^{-1}<\cdots$.

The method of proving arguments involving the forcing relation gives more information than just whether one cycle forces another. It also tells us how the two periodic orbits must be interlaced, that is, it gives us a permutation consisting of two cycles. The following example illustrates this. 
EXAMPLE 4.2. The cycle (123) forces the cycle (1). Looking at the map $I_{(123)}$, it can be seen that the fixed point must occur between the second and third points of the periodic orbit corresponding to (123). Thus, instead of writing (123) forces (1), we could include the additional information and write (123) forces (124) (3). Similarly, the fact that (123) forces (12) can be rewritten as (123) forces (135) (24). The cycle (132) forces (1) and (12) can be rewritten as (132) forces (143)(2) and (153)(24), respectively.

If $\alpha$ forces $\beta$, then there exists a periodic orbit of type $\beta$ in $I_{\alpha}$. The orbits of the points corresponding to $\alpha$ and $\beta$ give a permutation consisting of two cycles. Since there may be more than one periodic orbit of cycle type $\beta$ in $I_{\alpha}$, there may be more than one permutation that can be obtained in this way. We let $P[\alpha, \beta]$ denote the set containing all these permutations consisting of two cycles.

Initially, we say that a time-symmetric cycle $\alpha$ symmetrically forces a timesymmetric cycle $\beta$ if $\alpha$ forces $\beta$ and $\sigma \theta \sigma=\theta^{-1}$ for some $\theta \in P[\alpha, \beta]$. (Later, this definition will be slightly weakened to include more cases.)

In the example above, it is straightforward to check that $\sigma_{4}(124)(3) \sigma_{4}=$ (143) (2) which is not equal to the inverse (142)(3). So (123) does not symmetrically force (1). However, $\sigma_{5}(135)(24) \sigma_{5}=(153)(24)=[(135)(24)]^{-1}$. So (123) does symmetrically force (12).

This is easily seen graphically. Graphically, time symmetry is symmetry about the diagonal that goes from top left to bottom right. Below is a sketch of the graph of the cycle (123) (see Figure 4.1). It is impossible to draw a graph of a continuous function that connects the three dots and also has a symmetric fixed point.

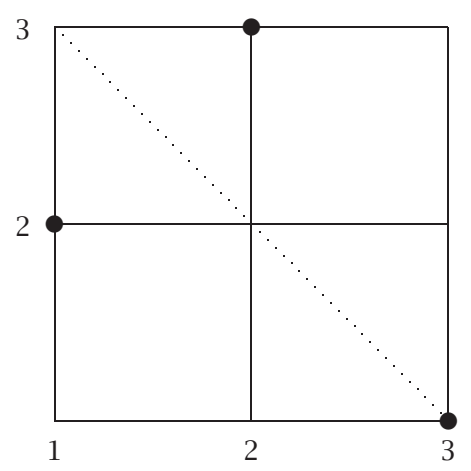

FIGURE 4.1

However, a graph can be drawn through these points that has a symmetric point of period two as indicated by the two new dots in Figure 4.2. 


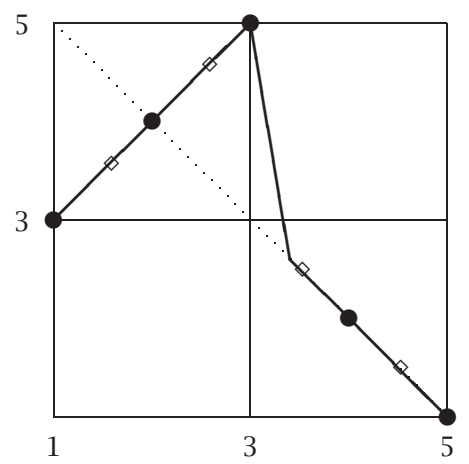

FIGURE 4.2

Now, (123) does force (1324) and $P[(123),(1324)]=(137)(2546)$ which is not symmetric. However, as indicated by the boxes in Figure 4.2 , it is possible to have a map of the interval with a periodic point of cycle type (1324) contained inside a periodic point of cycle type (123) in a symmetric way. We modify our definition of symmetric forcing to allow for this situation. The idea is that $\alpha$ should symmetrically force $\beta$ if they are both time symmetric, if $\alpha$ forces $\beta$, and if it is possible that both $\alpha$ and $\beta$ can be entwined in a time-symmetric way.

Suppose that we have a cycle $\beta$ of length $m$ and a cycle $\alpha$ of length $n$. Suppose that there is a permutation $\theta$ of length $n+m$ such that $\{1,2, \ldots, n+m\}$ consists of two orbits under $L_{\theta}$, one of type $\alpha$ and one of type $\beta$, where $L_{\theta}$ is the "connect the dot" map defined in Section 2 for cycles extended in the obvious way to permutations. If this can be done such that the endpoints 1 and $n+m$ and all the critical points of $L_{\theta}$ belong to the periodic orbit of cycle type $\alpha$, then we say that $\beta$ is contained within $\alpha$. Let $Q[\alpha, \beta]$ denote the set of permutations $\theta$ that consist of two cycles, one of type $\alpha$ and the other of type $\beta$, with $\beta$ contained within $\alpha$.

The following lemma follows directly from [1, Theorem 2.7.7].

LEMMA 4.3. Let $\alpha$ and $\beta$ denote two cycles with $\alpha \neq \beta$. Then, $\alpha$ forces $\beta$ if and only if $\beta$ is contained within $\alpha$. If $P[\alpha, \beta] \neq Q[\alpha, \beta]$, then $\beta$ is a double.

We say that a time-symmetric cycle $\alpha$ symmetrically forces a time-symmetric cycle $\beta$ if $\beta$ is contained within $\alpha$ and there exists a $\theta \in Q[\alpha, \beta]$ such that $\sigma \theta \sigma=\theta^{-1}$.

Returning to the example above, we have

$$
Q[(123),(1324)]=\{(137)(2546),(147)(2536)\} .
$$

Since $\sigma(147)(2536) \sigma=[(147)(2536)]^{-1}$, we obtain that (123) symmetrically forces (1324). 
It should be observed that the symmetric forcing is not a transitive relation: (123) symmetrically forces (12) and (12) symmetrically forces (1), but (123) does not symmetrically force (1). However, we have the following theorem.

THEOREM 4.4. Suppose that $\alpha$ and $\beta$ are unimodal time-symmetric cycles such that $\alpha<\beta$. Suppose that $\alpha$ and $\beta$ are consecutive in the sense that there is no time-symmetric $\gamma$ such that $\alpha<\gamma<\beta$. Then, $\beta$ symmetrically forces $\alpha$.

Proof. There are two cases to consider depending on whether $\alpha=[n]$ or $\alpha=[2 * n]$ for some integer $n$.

If $\alpha=[n]$, then the permutation

$$
(147 \cdots 3 n-2)(258 \cdots(3 n-4) 369 \cdots(3 n-3))
$$

shows that $[n]$ symmetrically forces $[2(n-1)]$.

If $\alpha=[2 * n]$, then the permutation

$$
(147 \cdots(3 n-2) 369 \cdots 3 n)(258 \cdots 3 n-1)
$$

shows that $[2 * n]$ symmetrically forces $[n]$.

In the next section, we consider symmetric forcing for nonconsecutive timesymmetric cycles.

5. A one-parameter family of maps. In this section, we introduce a oneparameter family of maps. As the parameter is varied, the "observable" behaviour goes through the sequence of periods given in Theorem 4.1. Intuitively, a periodic point is observable if its orbit is relatively unchanged under a small perturbation. More formally, an orbit of $a \in I$ given by $\left\{f^{n}(a) \mid n \geq 0\right\}$ is observable if, given any $\epsilon>0$, there exists a $\delta>0$ such that if $|x-a|<\delta$ then $\left|f^{n}(x)-f^{n}(a)\right|<\epsilon$ for any nonnegative integer $n$. Thus, points close to $a$ will $\epsilon$-shadow the orbit of $a$ for all time.

First, we study the one-parameter family $g_{r}(x): I \rightarrow I$ for $0<r<1$ defined by

$$
g_{r}(x)= \begin{cases}x+r & \text { if } 0 \leq x \leq 1-r \\ 1-x & \text { if } 1-r<x \leq 1\end{cases}
$$

These maps are time-symmetric in the sense that they have an inverse and $g_{r}^{-1}(x)=(1-x) \circ g_{r} \circ(1-x)$, but they are not continuous. Later, these maps will be modified on a small interval to make them continuous. The reason for studying $g_{r}$ is that the dynamics are simple and will give an important component of the dynamics of the modified maps.

The following lemma is easily seen to be true.

LEMmA 5.1. Suppose that $r=1 / n$ for some integer $n>1$. Then, $\{0,1 / n$, $2 / n, \ldots, 1\}$ is a periodic point of type $[n+1]$. For $0 \leq i \leq n-2$, the interval 
$(i / n,(i+1) / n)$ is linearly mapped onto the interval $((i+1) / n,(i+2) / n)$ in an orientation-preserving manner. The interval $((n-1) / n, 1)$ is linearly mapped onto $(0,1 / n)$ in an orientation-reversing manner.

Thus, if $r=1 / n$ for some integer $n>1$, every point in the unit interval is periodic. There is an orbit of type $[n+1]$ and one of type $[n]$, and every other point belongs to an orbit of type [2*n].

It is straightforward to check the following.

LEMMA 5.2. Suppose that $1 /(n+1)<r<1 / n$ for some integer $n>1$. Then, for $0 \leq i \leq n$, the interval [ir, $1-n r+i r$ ] is linearly mapped in an orientationpreserving way onto $[(i+1) r, 1-n r+(i+1) r]$ and the interval $[n r, 1]$ is linearly mapped onto $[0,1-n r]$ in an orientation-reversing way. For $1 \leq i \leq$ $n-1$, the interval $(1-n r+(i+1) r$,ir $)$ is linearly mapped in an orientationpreserving way onto $(1-n r+(i+2) r,(i+1) r)$ and $(1-r, n r)$ is mapped onto $(1-n r, r)$ in an orientation-reversing way.

Thus, if $1 /(n+1)<r<1 / n$, every point in the interval is periodic. There is one periodic orbit of type $[n]$, one of type $[n+1]$, and every other point belongs to orbits of type $[2 * n]$ and $[2 *(n+1)]$. The widths of the intervals mentioned in the above lemma are $1-n r$ for the closed intervals containing points in the orbits of type $[2 *(n+1)]$ and midpoint of type $[n+1]$, and $(n+1) r-1$ for the open intervals containing the points in the orbits of type [2*n] with midpoints of type $[n]$. Thus, as $r$ changes from $1 / n$ to $1 /(n+1)$, the closed intervals expand and the open intervals contract. When $r=1 /(n+1)$, the open intervals disappear and the endpoints of what were the closed intervals give a new periodic point of type $[n+2]$. As $r$ is further decreased, the points in the orbit expand into closed intervals with midpoints of type $[n+2]$ and surrounding points of type $[2 *(n+2)]$. It is clear that all points in the interior of these intervals belong to observable orbits.

As noted above, the maps $g_{r}$ are not continuous. Since the study of onedimensional combinatorial dynamics relies heavily on the intermediate value property, we will be slightly modifying the maps to make them continuous. This modification will of necessity mean that they will no longer be invertible. However, there are intervals corresponding to the intervals in the example above and, if we restrict to these intervals, then the maps are invertible. Moreover, the dynamics of the periodic points that are outside these intervals is not observable.

Define $f_{\mu, r}(x)$ for $0<r<1$ and $0<\mu<r$ by

$$
f_{\mu, r}(x)= \begin{cases}x+r & \text { if } 0 \leq x \leq 1-r, \\ \left(\frac{r-1-\mu}{\mu}\right) x+\frac{(1-r)(1-r+\mu)+\mu}{\mu} & \text { if } 1-r<x<1-r+\mu, \\ 1-x & \text { if } 1-r+\mu \leq x \leq 1 .\end{cases}
$$


The following two lemmas are easily seen to be true.

LEMMA 5.3. If $\mu<(1-r) / 2$ and if $a \in(1-r, 1-r+\mu)$ is periodic under $f_{\mu, r}$, then the orbit of $a$ is not observable.

LEMMA 5.4. Let $1 /(n+1) \leq r \leq 1 / n$. If $\mu<1 / 2(n+1)$, then there exist periodic orbits that never enter the open interval $(1-r, 1-r+\mu)$.

We can define a one-parameter family of continuous maps of the interval $h_{r}$ by $h_{r}(x)=f_{\mu, r}(x)$ where $\mu$ is taken to be the minimum of $(1-r) / 3$ and $r / 3(r+1)$. This choice of $\mu$ satisfies the conditions in the previous two lemmas and then the proof of the following theorem is straightforward.

THEOREM 5.5. Let $h_{r}$ be the one-parameter family of continuous maps of the interval defined above. Let $n$ denote a positive integer.

When $r=1 / n$, there exist observable periodic orbits of types $[n]$ and [2*n], and these are the only observable periodic orbits.

When $(2 \mu+1) /(n+1)<r<1 / n$, there exist observable periodic orbits of types $[n],[2 * n],[n+1]$, and $[2 *(n+1)]$, and these are the only observable periodic orbits.

When $1 /(n+1)<r \leq(2 \mu+1) /(n+1)$, there exist observable periodic orbits of types $[n+1]$ and $[2 *(n+1)]$, and these are the only observable periodic orbits.

Thus, as $r$ goes from 1 to 0 , a periodic point of type $[N+1]$ is formed when $r=1 / n$. Initially, this orbit is not observable, but, as $r$ decreases below $1 / n$, it becomes observable and is surrounded by points of type $[2 *(n+1)]$. These orbits persist until $r$ reaches $(2 \mu+1) /(n+2)$.

6. Comment. In this paper, we restricted attention to the unimodal case because of the ease of giving a complete classification. In many physical systems as a parameter is changed, a sequence of period doublings has been observed. As shown above, in the time-symmetric unimodal case, this cannot occur. However, if we drop the unimodal requirement, it is possible to find a time-symmetric double of any time-symmetric cycle. However, as we move through a doubling sequence, the modality of the underlying map increases (see [3]).

\section{REFERENCES}

[1] L. Alsedà, J. Llibre, and M. Misiurewicz, Combinatorial Dynamics and Entropy in Dimension One, Advanced Series in Nonlinear Dynamics, vol. 5, World Scientific Publishing, New Jersey, 2000.

[2] S. Baldwin, Generalizations of a theorem of Sarkovskii on orbits of continuous realvalued functions, Discrete Math. 67 (1987), no. 2, 111-127.

[3] C. Bernhardt, Time-symmetric cycles, to appear in Int. J. Math. Math. Sci.

[4] - The ordering on permutations induced by continuous maps of the real line, Ergodic Theory Dynam. Systems 7 (1987), no. 2, 155-160. 
[5] L. S. Block and W. A. Coppel, Dynamics in One Dimension, Lecture Notes in Mathematics, vol. 1513, Springer-Verlag, Berlin, 1992.

[6] R. Shaw, The Dripping Faucet as a Model Chaotic System, The Science Frontier Express Series, Aerial Press, California, 1984.

Chris Bernhardt: Department of Mathematics and Computer Science, Fairfield University, Fairfield, CT 06824, USA

E-mail address: cbernhardt@fair1.fairfie1d.edu 


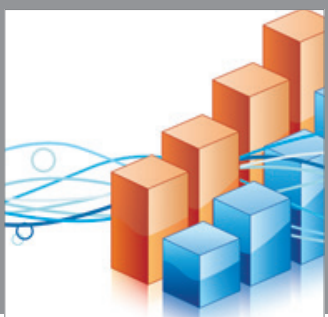

Advances in

Operations Research

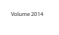

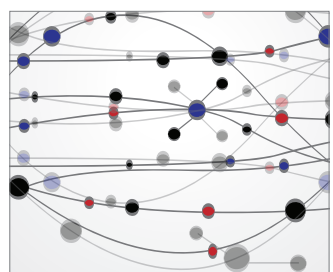

\section{The Scientific} World Journal
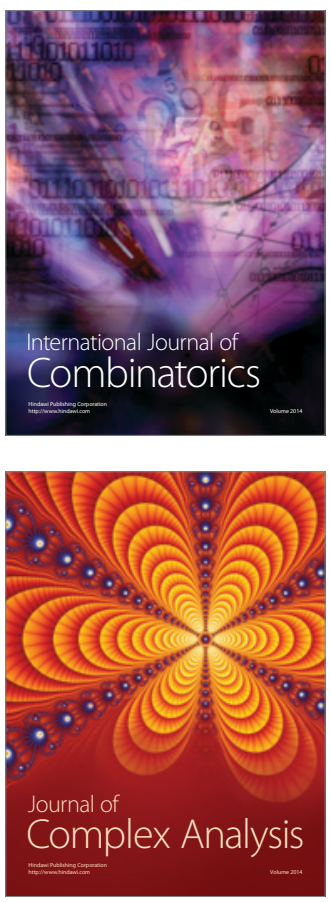

International Journal of

Mathematics and

Mathematical

Sciences
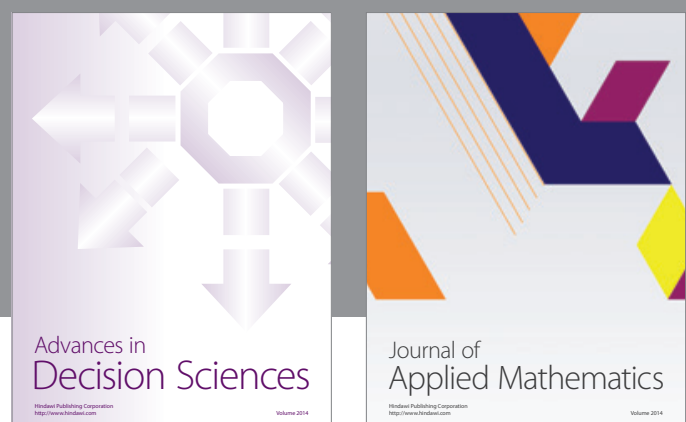

Journal of

Applied Mathematics
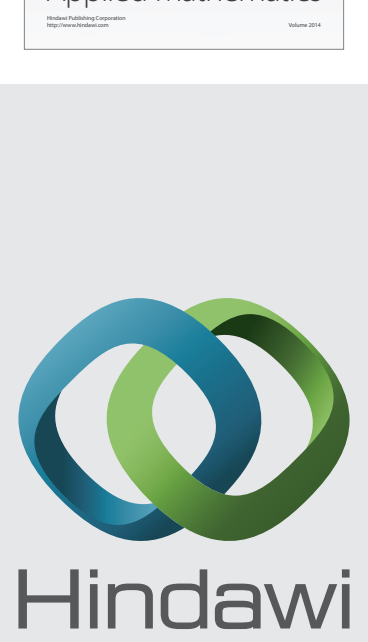

Submit your manuscripts at http://www.hindawi.com
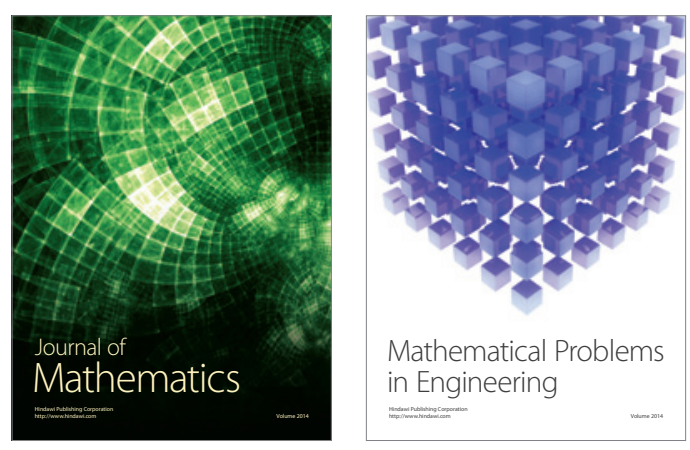

Mathematical Problems in Engineering
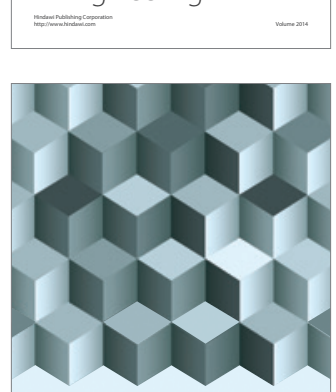

Journal of

Function Spaces
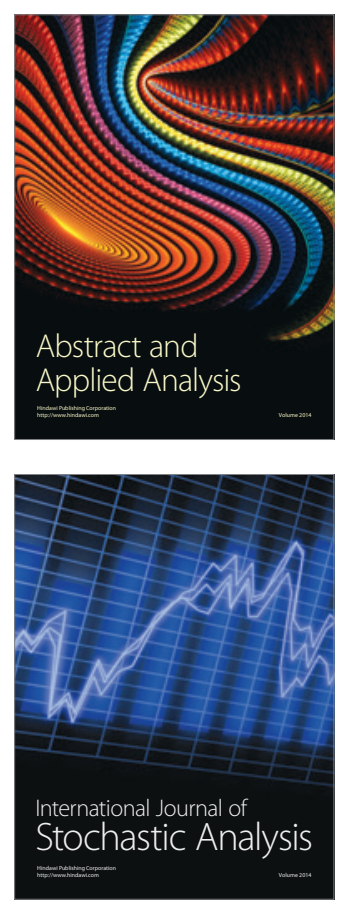

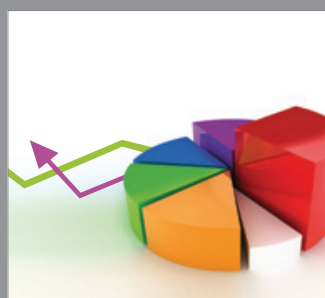

ournal of

Probability and Statistics

Promensencen
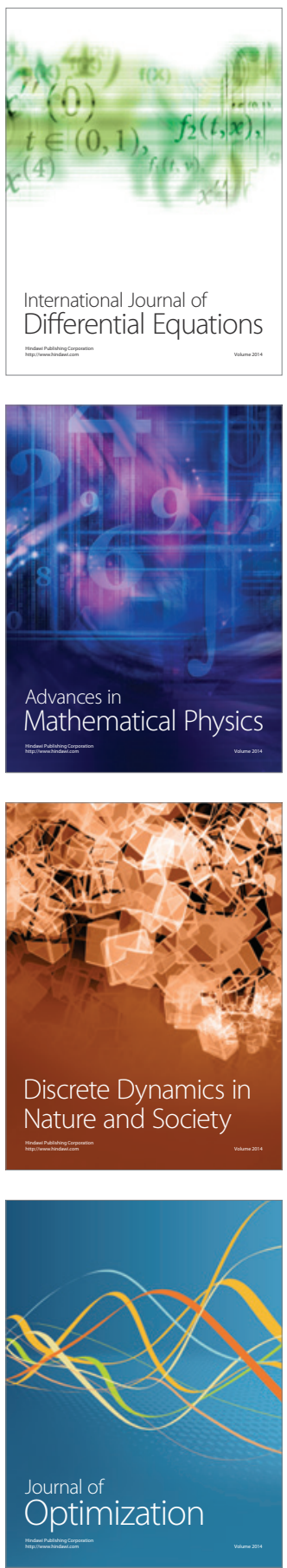